\title{
Describing Urban Evolution with the Fractal Parameters Based on Area-Perimeter Allometry
}

\author{
Yanguang Chen ${ }^{1}$ and Jiejing Wang ${ }^{2}$ \\ ${ }^{1}$ Department of Geography, College of Urban and Environmental Sciences, Peking University, Beijing 100871, China \\ ${ }^{2}$ Department of Urban Planning and Design, The University of Hong Kong, Pokfulam Road, Pokfulam, Hong Kong \\ Correspondence should be addressed to Jiejing Wang; jackiewang1120@gmail.com
}

Received 15 October 2015; Revised 10 December 2015; Accepted 10 December 2015

Academic Editor: Vicenç Méndez

Copyright (c) 2016 Y. Chen and J. Wang. This is an open access article distributed under the Creative Commons Attribution License, which permits unrestricted use, distribution, and reproduction in any medium, provided the original work is properly cited.

\begin{abstract}
The area-perimeter allometric scaling is an important approach for researching fractal cities, and the basic ideas and models have been researched for a long time. However, the fractal parameters based on this scaling relation have not been efficiently utilized in urban studies. This paper is devoted to developing a description method of urban evolution using the fractal parameter sets based on the area-perimeter measure relation. The novelty of this methodology is as follows: first, the form dimension and boundary dimension are integrated to characterize the urban structure and texture; second, the global and local parameters are combined to characterize an urban system and individual cities; third, an entire analytical process based on the area-perimeter scaling is illustrated. Two discoveries are made in this work: first, a dynamic proportionality factor can be employed to estimate the local boundary dimension; second, the average values of the local fractal parameters are approximately equal to the corresponding global fractal parameters of cities. By illustrating how to carry out the area-perimeter scaling analysis of Chinese cities in Yangtze River Delta in the case of remote sensing images with low resolution, we propose a possible new approach to exploring fractal systems of cities.
\end{abstract}

\section{Introduction}

A scientific study is always involved with two processes: the first process is to describe how a system works using mathematics or measurement, and the second process is to gain understanding of the causality behind the system using observation, experience, or artificially constructed experiments $[1,2]$. Fractal geometry is a powerful tool of describing urban systems because fractal dimension is a kind of characteristic parameter to describe scale-free phenomena [3-9]. By fractal dimension description, we can get insight into the spatial dynamics of urban evolution [10-13]. There are two simple and convenient ways of understanding city fractals. One is measurement process (see, e.g., [14-19]), and the other is the geometric measure relation (see, e.g., [12, $20,21]$ ). If a measurement result such as boundary length, urban area, and land-use density depends on the scale to measure (the length of yardstick), we may face a fractal object. On the other hand, if the proportional relation between two measures such as urban area and urban perimeter is involved with a scaling exponent indicating a pair of fractional parameters, we will meet a fractal phenomenon. The former way can be used to realize any types of fractals, while the latter way can be employed to identify the fractal boundary of a region. Sometimes an urbanized area is treated as a Euclidean space, but the urban boundary can be regarded as a fractal line. In this case, the boundary dimension of a city provides a good way of looking at city size and shape [4]. So far, we have more than five empirical approaches to estimating the fractal dimension of geographical boundaries [22-27].

An urban area bears an analogy to a random Koch island; thus, the fractal dimension of its boundary can be estimated with the area-perimeter scaling $[4,22,28,29]$. The geometrical measure relation between urban area and perimeter is in essence an allometric scaling relation, which is similar to the relation between urban area and population [30]. If the population size of a city is compared to the weight of an animal, then the urban area can be compared to the volume of 
the animal and the urban perimeter to the surface area (the area of the whole skin) [20]. In many cases, it is difficult to investigate city population, but it is easy to measure urban area and the corresponding perimeter by using the remote sensing images and the geographical information system (GIS) techniques. The area-perimeter allometric scaling is a simple approach to revealing the spatiotemporal evolution dynamics of urban systems. However, two problems remain to be solved. First, in many cases, it is impossible to compute the fractal dimension of each city in an urban system because of inadequate remote sensing data or the lower resolution of remote sensing images. Second, the traditional formula of the boundary dimension based on the area-perimeter scaling is not exact enough to guarantee the effective results.

The processes and patterns of urban evolution follow scaling laws [31-34]. Fractal geometry is an effective tool to explore scaling in cities. In recent years, the new formulae for revising the boundary dimension calculations through the area-perimeter scaling have been proposed [23]. By means of these formulae, we can correct the errors of the boundary dimension and estimate the form dimension of cities. Using the boundary dimension and form dimension, we can characterize urban shapes and the spatiotemporal evolution of urban systems. This paper is devoted to developing the description method of urban patterns and processes using the fractal dimension sets based on the areaperimeter allometric scaling. It tries to solve several problems as follows: how to combine the global fractal parameters with the local fractal parameters for spatial analysis; how to make use of remote sensing images of low resolution for urban fractal studies; how to understand the influence of urban sprawl on fractal dimension change. The rest of the work is organized as follows. In Section 2, several new formulae of fractal dimension estimation are introduced and clarified for spatiotemporal analysis of urban form and growth. In Section 3, the new models and formulae are applied to the system of cities and towns in Yangtze River Delta, China, to make an empirical analysis. In Section 4, based on the mathematical models and the empirical case, the new analytical process of urban evolution based on the urban area-perimeter scaling is presented and illustrated, and several related questions are discussed. Finally, the paper reaches its conclusions by outlining its major viewpoints.

\section{Fractal Parameters}

2.1. Basic Formulae. In theory, a city figure can be divided into two parts: one is the urban boundary, and the other is the urban area within the boundary. The former is termed urban envelope $(E)$ and can be described with the boundary dimension [21], while the latter is named urban area $(A)$ and can be characterized by the form dimension $[4,35]$. The form dimension is a structural dimension, while the boundary dimension is a textural dimension $[20,36,37]$. The form dimension and boundary dimension compose the shape dimension of cities. In fact, the family of shape indexes includes the well-known form ratio, which is defined by area and perimeter of a city [38]. The form dimension can be measured by the area-scale relation, while the boundary dimension can be determined by the perimeter-scale relation [23]. In this sense, urban shape can be characterized by both the form and the boundary dimensions.

Based on digital maps or remote sensing images, fractal cities are always defined in a 2-dimensional space. In practice, the urban area can be regarded as a Euclidean plane with a dimension $d=2$, and accordingly, the urban boundary is treated as a fractal line $[4,23]$. Thus, the boundary dimension represents the basic fractal dimension of urban shape and can be estimated with the regression analysis based on the method of the ordinary least squares (OLS). By the fractal measure relation $[28,29,39]$, the urban area and perimeter follow a power law as below:

$$
\left(\frac{P}{k}\right)^{1 / D_{l}}=A^{1 / 2},
$$

where $P$ refers to the perimeter of the urban envelope and $A$ refers to the corresponding urban area. As for the parameters, $D_{l}$ denotes the boundary dimension of traditional meaning and $k$ is related with the proportionality coefficient. In this context, the boundary dimension $D_{l}$ should be termed initial boundary dimension because it represents the conventional concept of the fractal dimension of urban boundary. Equation (1) is in fact an allometric scaling relation, and the scaling exponent is

$$
b=\frac{D_{l}}{d}=\frac{D_{l}}{2},
$$

where $d=2$ denotes the Euclidean dimension of the embedding space of urban form. The boundary dimension is often estimated by the formula $D_{l}=2 b$. From (1), it follows that

$$
D_{l}=\frac{2 \ln (P / k)}{\ln (A)},
$$

which is an approximate formula of the boundary dimension estimation. By analogy with squares and empirical analysis, the proportionality parameter is always taken as fixed value; that is, $k=4[40,41]$. In fact, the fixed coefficient can be replaced by a dynamic parameter, which can be estimated with regression analysis. Thus, (2) provides a simple approach to estimating the boundary dimension especially when spatial data are short for computing the fractal dimension.

2.2. Adjusting Formulae. It is easy to evaluate the boundary dimension of a fractal region such as the Koch island and urban envelope [21,36]. However, the method based on the geometric measure relation always overestimates the fractal dimension value $[22,30]$. In order to lessen the errors resulting from (1) and (2), a formula is derived as follows [23]:

$$
D_{b}=\frac{1+D_{l}}{2},
$$

where $D_{b}$ represents the revised boundary dimension of a city (a textural dimension). Equation (4) suggests a linear relation between $D_{l}$ and $D_{b}$. If we calculate the value of $D_{l}$ using (3) or log-linear regression analysis, we can estimate the $D_{b}$ value by means of (4). 
In reality, a city is a complex spatial system, and urban area does not correspond to a 2-dimensional region. In this case, (1) is not enough to describe the geometric measure relation between urban area and perimeter. According to the studies of Cheng [42], Imre [43], and Imre and Bogaert [44], (1) can be generalized as follows:

$$
(k P)^{1 / D_{b}}=A^{1 / D_{f}},
$$

where $D_{f}$ denotes the fractal dimension of urban form within the urban envelope (a structural dimension). The form dimension $D_{f}$ can be estimated with the following formula [23]:

$$
D_{f}=1+\frac{1}{D_{l}},
$$

which suggests a hyperbolic relation between $D_{l}$ and $D_{f}$. From (4) and (6), it follows that

$$
\frac{1}{D_{f}}=1-\frac{1}{2 D_{b}},
$$

which suggests a hyperbolic relation between $D_{b}$ and $D_{f}$. Accordingly, $1 / D_{b}=2-2 / D_{f}$. If $D_{b}=1$, then we have $D_{f}=2$, and vice versa. If so, we will have a Euclidean object. Based on (5), the allometric scaling exponent expressed by (2) can be rewritten as

$$
\sigma=\frac{D_{b}}{D_{f}}
$$

where $\sigma$ refers to the revised scaling exponent of the areaperimeter allometry. An allometric exponent is usually a ratio of one fractal dimension to another fractal dimension. In many cases, it is allometric scaling exponents rather than fractal dimensions that play an important role in spatial analysis of urban systems $[30,45,46]$.

Using the mathematical models, allometric scaling relations, and the fractal parameter formulae, we can describe and analyze the spatial development and evolution of urban systems in the real world. For an urban system, the fractal dimensions and the related allometric scaling exponents can be classified as global fractal parameters and local fractal parameters. The global fractal parameters can be reckoned with the cross-sectional data and used to describe a system of cities as a whole, while the local fractal parameters can be figured out by using the data of individual cities and used to describe each city as an element in the urban system.

\section{Empirical Analysis}

3.1. Study Area and Method. The area-perimeter scaling and the adjusting formulae of fractal dimension can be applied to the actual cities by means of remote sensing data. Yangtze River Delta in China is taken as a study area to make an empirical analysis. The region includes 68 cities and towns, which can be regarded as an urban system (Figure 1). The remote sensing images used in this research came from National Aeronautics and Space Administration (NASA), including Landsat MSS, TM, and ETM images from 1985, 1996, and 2005, which were downloaded from the Earth Science Data Interface (ESDI) at the Global Land Cover Facility (GLCF) center of University of Maryland (http://glcf.umd.edu/). Firstly, these images were transformed to the GCS_WGS_1984 geographic coordinate system and Asia_Lambert_Conformal_Conic projected coordinate system. Then, the supervised classification method (SCM) was employed to extract the built-up area and the boundary of each city in a given year. The results can be manually corrected in ESRI ArcGIS to ensure better accuracy. The SCM is one of the popular remote sensing image classification techniques [47]. The basic idea of this technique is to select "training areas" as representative samples to identify the land cover classes in an image. The classifier is then used to attach labels to all the image pixels according to the trained parameters. The maximum likelihood classification (MLC) is commonly used to generate the trained parameters, such as mean vectors and variance-covariance matrix. Then, the land cover is classified based on the spectral signature that is defined by these trained parameters. In this work, we use the software of ERDAS IMAGINE which has a well-defined classifier for the SCM. We classify the Landsat images into five classes of land cover: city, forest, agriculture, water, and other. The land cover of city can be obtained and then the rough boundary of cities can be extracted on the base of the classification.

The next step is the manual error correction of the land cover of cities obtained in the first step. We check the boundary by comparing with the existing maps. The Google Map and Chinese Administrative Division Maps for various years are the major sources for manual error correction. The classified land cover of city is geocoded with these maps. So we can drop the pixels which are not located within the city administrative boundary. Afterwards, we can use a range of critical landmark features (e.g., river, lake, and mountain) as benchmarks to correct the outlier pixels and the pixels that are wrongly classified. Based on the error correction, the much more accurate city boundary and urban land use can be extracted in ArcGIS. As such, we can extract the accurate built-up area which falls into each urban envelope, and then calculate areal and perimetric values through the Calculate Geometry function in the Attribute Table of ArcGIS. Finally, we have 3 datasets of urban areas and perimeters for the 68 cities and towns in three years (Table 1).

Using the relations and formulae, we can evaluate the scaling exponents and fractal dimensions. If the observational data have been processed, the procedure of parameter estimation is as follows.

Step 1 (preliminary estimation of fractal parameters). Using the geometric measure relation and formulae including (1) and (2), we can calculate the initial boundary dimension $D_{l}$ and the proportionality coefficient $k$ by means of scatterplots and the least squares regression.

Step 2 (revision of the global fractal parameters). Using (4) and (6), we can transform the initial global boundary dimension $D_{l}$ into the revised global boundary dimension $D_{b}$ and global form dimension $D_{f}$, which are used to describe the urban system in the study area. 


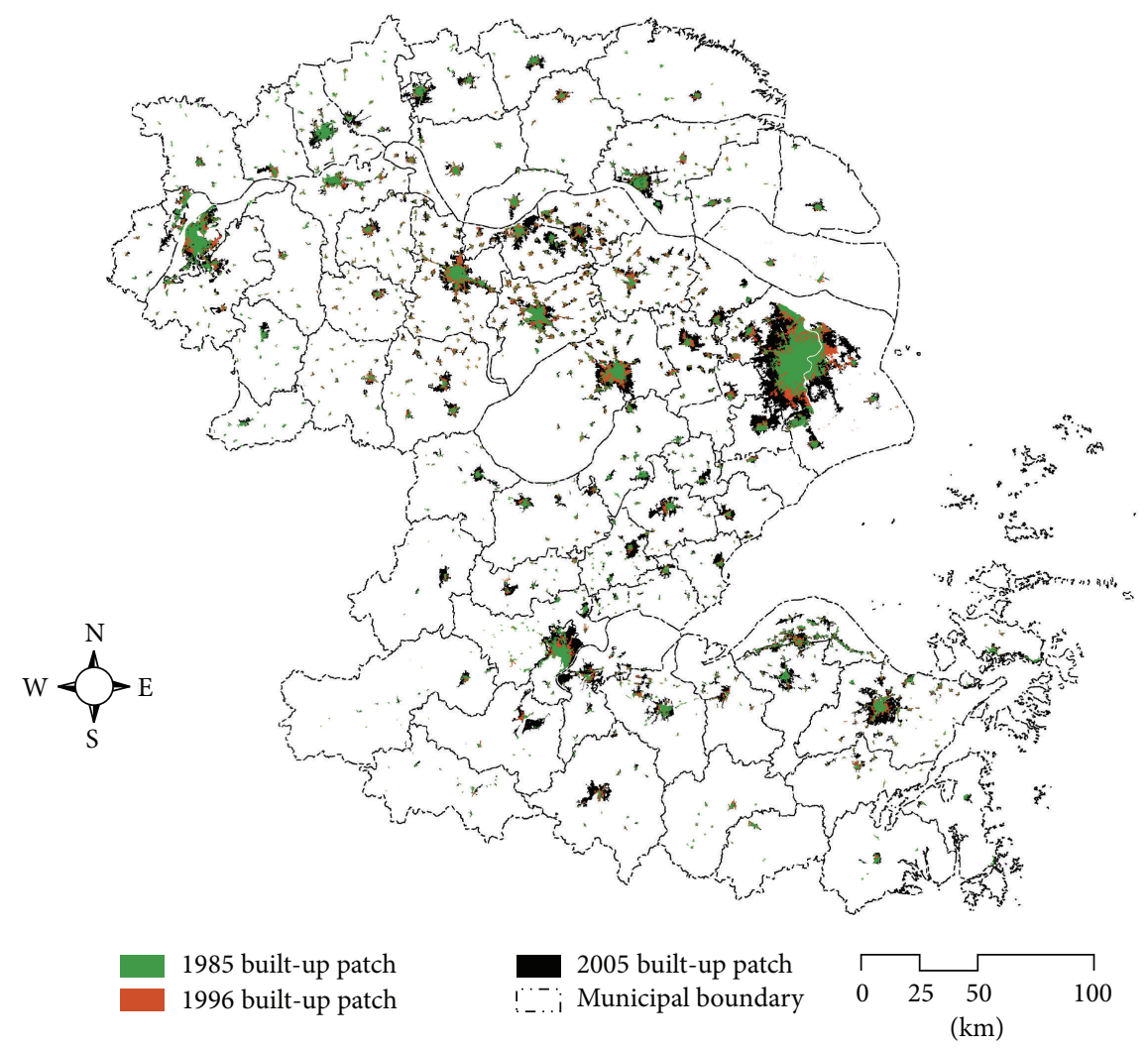

FIGURE 1: A sketch map of the 68 cities and towns in Yangtze River Delta, China (1985-2005).

Step 3 (estimation of the local fractal parameters). Using the approximate formula, (3), and the $k$ values from Step 1, we can estimate the initial local boundary dimension $D_{l}$ for each city.

Step 4 (revision of the local fractal parameters). Using (4) and (6), we can transform the initial local boundary dimension $D_{l}$ into the revised local boundary dimension $D_{b}$ and local form dimension $D_{f}$, which are used to describe the individual cities in the study area.

Step 5 (related calculations). Fractal parameters can be associated with traditional spatial measurements. For example, the local boundary dimension is a function of the compactness of urban form. The compactness ratio of a city can be figured out with urban area and perimeter.

3.2. Results. The regression analysis based on OLS can be employed to estimate the allometric parameters and the global fractal parameters. The log-log scatterplots show that the relations between urban area and perimeter follow the allometric scaling law (Figure 2). The slopes of the trend lines give the values of the scaling exponent $b$ in different years. Taking natural logarithms on both sides of (1) yields a linear relation as below:

$$
\ln P=\ln k+\frac{D_{l}}{2} \ln A=C+b \ln A
$$

where $C=\ln k$ and $b=D_{l} / 2$. Using the least squares calculation, we can fit (9) to the datasets displayed in Table 1 . The results including the values of the proportionality coefficient $k$, scaling exponent $b$, and the goodness of fit $R^{2}$ are tabulated as below (Table 2). By (2), (4), and (6), we can further estimate the initial boundary dimension $D_{l}$, the revised boundary dimension $D_{b}$, and the form dimension $D_{f}$. The values of boundary dimension $D_{l}$ range from 1.4 to 1.5. Accordingly, the revised boundary dimension $D_{b}$ is about 1.2, and the corresponding form dimension $D_{f}$ is around 1.7.

If we had the remote sensing images with enough high resolution of all the cities, we would calculate the form and boundary dimension for each city $[16,27]$. If so, the local fractal parameters could be evaluated with the regression analysis or other algorithms such as the major axis method (MAM). Unfortunately, we have only the images of the region instead of each city. In this case, the local parameters can be estimated by (3). The key is to determine the value of $k$. There are two possible approaches to estimating the $k$ value. One is empirical method. As indicated above, a fixed coefficient $k=4$ is proposed by empirical analysis $[40,41]$. However, if we take $k=4$, the boundary dimension of the cities in our study area will be significantly overestimated. For example, for 1985, the average $D_{l}$ value of the 68 cities and towns is about 2.258, which is greater than the Euclidean dimension of the embedding space and thus unreasonable. Generally speaking, the boundary dimension comes between 1 and 1.5, 


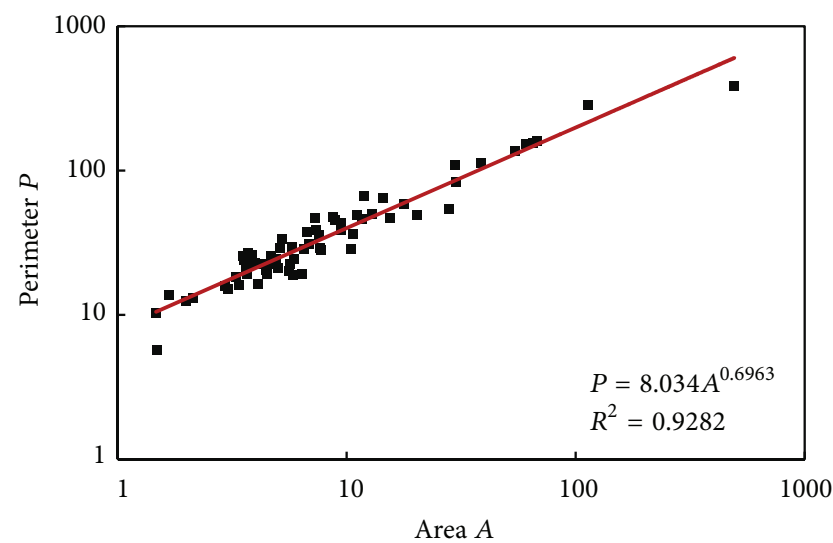

(a) 1985

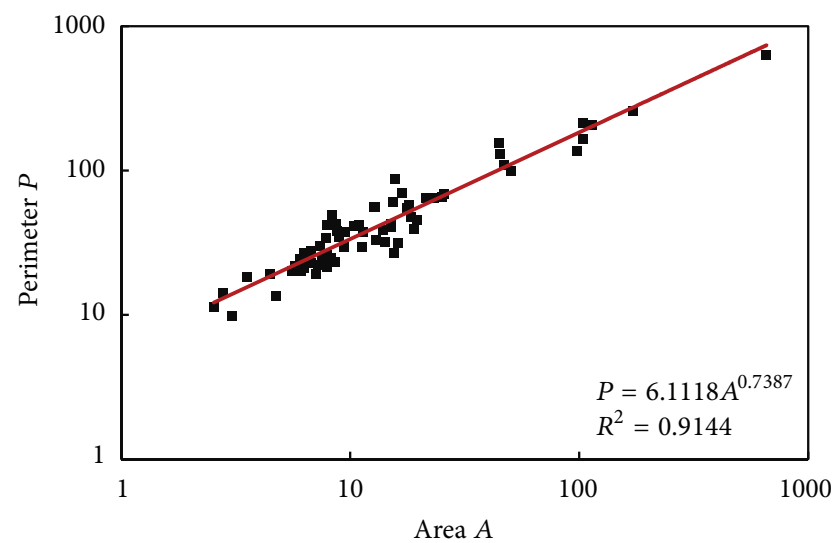

(b) 1996

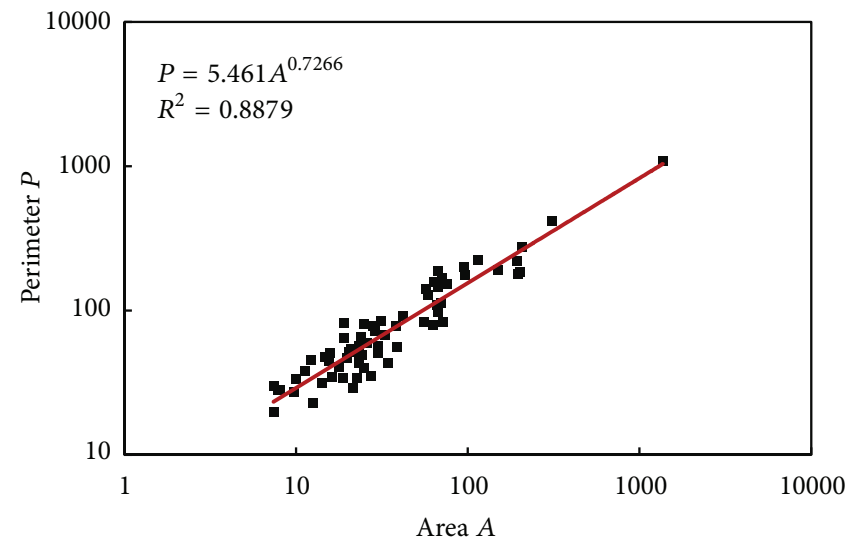

(c) 2005

FIGURE 2: The allometric scaling relations between urban area and perimeter of the cities and towns in Yangtze River Delta, China (1985-2005).

and the average value is often near 1.25 [20, 30, 48]. The other is the regression method. By the least squares calculation of cross-sectional datasets, we can estimate the $k$ values using (9). This is an unfixed parameter: the $k$ value changes over time (Table 2). Using the variable $k$ value to replace the fixed $k$ value in (3), we can estimate the boundary dimension $D_{l}$ of each city. Then, using (4) and (6), we can further estimate the revised boundary dimension $D_{b}$ and the corresponding form dimension $D_{f}$ for each city. All these results represent the local fractal parameters of the urban system in the study region (Table 3 ).

A discovery is that the average values of the local fractal parameters are approximately equal to the corresponding global fractal parameters of the whole cities and towns. Comparing the means of the fractal dimensions listed in Table 3 with the related parameter values displayed in Table 2, we can find the consistency of the global estimation with the local average (Table 4 ). The global parameter values can be used to analyze the development of the system of cities and towns at the macrolevel (the whole), while the local parameter values can be employed to analyze the evolution process of the individual cities at the microlevel (the parts or elements).

3.3. Global and Local Analysis. Generally speaking, a mathematical model reflects the macrostructural properties of a system, while the model parameters reflect the microinteraction of elements. It is necessary to examine the mathematical expressions of area-perimeter relations and the corresponding fractal parameters. Analyzing the model forms and parameter values will result in a number of findings.

First, the allometric scaling degenerated from power law to linear relations from 1985 to 2005 . A real allometric scaling relation takes on a double logarithmic relation, but it sometimes degenerates and changes to single logarithmic relations including exponential relation and logarithmic relation (semidegeneration), or even to a linear relation (full degeneration) [49]. In 1985, the area-perimeter relation followed the power law, and the goodness of fit $\left(R^{2}\right)$ of the power function was significantly higher than those of the linear function and the single logarithmic functions. However, in 1996, the case was different, and the goodness of fit of the linear function is a little higher than that of the power function. In 2005, the goodness of fit of the linear function is significantly higher than that of the power function (Table 5). A complex system such as a city and a system of cities can be modeled by more than one mathematical form. Different mathematical models reflect different states of system evolution. Fractals suggest the optimized structure of nature. In theory, fractal models represent the optimum state of city development as a fractal object can fill its space in the best way. The degeneration of 
TABLE 1: The urban area and perimeter datasets of the cities and towns in Yangtze River Delta, China (1985-2005).

\begin{tabular}{|c|c|c|c|c|c|c|}
\hline \multirow{2}{*}{ City/town } & \multicolumn{2}{|c|}{1985} & \multicolumn{2}{|c|}{1996} & \multicolumn{2}{|c|}{2005} \\
\hline & Area $A$ & Perimeter $P$ & Area $A$ & Perimeter $P$ & Area $A$ & Perimeter $P$ \\
\hline Shanghai & 492.52 & 383.30 & 657.73 & 629.58 & 1368.11 & 1095.44 \\
\hline Qingpu & 5.23 & 33.56 & 9.36 & 29.78 & 25.93 & 59.29 \\
\hline Chongming & 4.89 & 24.51 & 6.29 & 22.85 & 9.69 & 27.51 \\
\hline Songjiang & 12.88 & 49.74 & 15.46 & 26.74 & 71.93 & 83.81 \\
\hline Jinshan & 4.43 & 20.53 & 5.72 & 21.83 & 27.36 & 35.15 \\
\hline Nanjing & 113.24 & 286.55 & 171.48 & 257.13 & 311.11 & 418.07 \\
\hline Jiangning & 7.72 & 28.23 & 17.62 & 57.84 & 41.85 & 91.62 \\
\hline Jiangpu & 3.70 & 26.97 & 8.29 & 49.04 & 15.84 & 50.94 \\
\hline Liuhe & 4.67 & 22.44 & 7.95 & 26.16 & 16.17 & 34.78 \\
\hline Lishui & 9.46 & 43.18 & 9.46 & 37.82 & 21.04 & 53.97 \\
\hline Gaochun & 4.47 & 19.26 & 4.47 & 19.26 & 11.32 & 37.98 \\
\hline Wuxi & 60.54 & 152.58 & 98.23 & 137.39 & 149.01 & 192.22 \\
\hline Jiangyin & 15.51 & 46.77 & 25.75 & 69.01 & 95.17 & 199.15 \\
\hline Yixing & 5.13 & 29.33 & 11.37 & 37.58 & 38.09 & 78.15 \\
\hline Changzhou & 64.98 & 156.09 & 114.00 & 207.79 & 205.62 & 275.72 \\
\hline Liyang & 3.79 & 22.46 & 12.71 & 56.49 & 18.95 & 64.27 \\
\hline Jintan & 4.69 & 25.72 & 10.91 & 41.98 & 19.69 & 46.87 \\
\hline Suzhou & 54.19 & 137.89 & 104.30 & 166.28 & 197.10 & 180.47 \\
\hline Changshu & 11.92 & 67.02 & 25.26 & 66.09 & 58.60 & 127.81 \\
\hline Zhangjiagang & 6.70 & 37.78 & 18.42 & 47.52 & 70.19 & 112.34 \\
\hline Kunshan & 8.85 & 45.19 & 18.92 & 39.67 & 62.45 & 79.94 \\
\hline Wujiang & 6.47 & 28.46 & 7.92 & 21.45 & 18.73 & 34.46 \\
\hline Taicang & 7.57 & 35.55 & 11.29 & 29.53 & 38.82 & 55.76 \\
\hline Nantong & 27.94 & 53.85 & 46.99 & 109.35 & 114.53 & 226.23 \\
\hline Hai'an & 10.70 & 36.20 & 12.99 & 32.86 & 29.97 & 57.21 \\
\hline Rudong & 4.09 & 16.44 & 7.06 & 19.32 & 14.23 & 31.38 \\
\hline Haimen & 5.81 & 19.05 & 7.55 & 25.01 & 15.53 & 44.87 \\
\hline Qidong & 6.35 & 19.33 & 8.57 & 23.42 & 20.26 & 51.45 \\
\hline Rugao & 5.74 & 29.75 & 15.42 & 60.47 & 23.25 & 53.01 \\
\hline Yangzhou & 38.53 & 112.91 & 44.59 & 154.97 & 75.57 & 154.09 \\
\hline Jiangdu & 7.60 & 29.05 & 10.36 & 41.29 & 31.05 & 85.12 \\
\hline Yizheng & 8.73 & 47.51 & 13.86 & 38.58 & 24.37 & 49.05 \\
\hline Taizhou & 17.80 & 58.73 & 21.51 & 64.12 & 70.61 & 169.03 \\
\hline Jiangyan (Tai) & 6.48 & 28.73 & 8.72 & 38.09 & 24.98 & 80.58 \\
\hline Taixing & 9.45 & 38.95 & 15.11 & 40.68 & 23.77 & 65.96 \\
\hline Jingjiang & 11.07 & 49.63 & 16.11 & 31.82 & 28.23 & 78.59 \\
\hline Zhenjiang & 29.67 & 109.18 & 44.83 & 130.62 & 67.51 & 186.67 \\
\hline Jurong & 3.54 & 25.73 & 6.74 & 27.58 & 10.06 & 33.42 \\
\hline Yangzhong & 3.65 & 19.13 & 6.28 & 21.64 & 12.25 & 45.72 \\
\hline Danyang & 7.33 & 38.86 & 15.09 & 42.77 & 33.02 & 67.58 \\
\hline Hangzhou & 67.49 & 160.88 & 103.41 & 213.64 & 200.66 & 184.77 \\
\hline Tonglu & 1.98 & 12.56 & 2.78 & 14.11 & 7.43 & 30.32 \\
\hline Fuyang & 2.94 & 15.79 & 7.82 & 34.39 & 55.75 & 83.71 \\
\hline Lin'an & 3.86 & 25.85 & 6.26 & 26.81 & 16.25 & 34.92 \\
\hline Yuhang & 1.47 & 10.33 & 6.04 & 24.35 & 17.88 & 40.72 \\
\hline Xiaoshan & 4.99 & 21.17 & 16.76 & 69.84 & 96.49 & 178.18 \\
\hline Ningbo & 29.93 & 82.98 & 50.54 & 99.57 & 193.81 & 219.05 \\
\hline Xiangshan & 3.62 & 21.26 & 2.54 & 11.42 & 7.39 & 20.01 \\
\hline Ninghai & 5.61 & 20.31 & 7.26 & 22.15 & 12.51 & 23.07 \\
\hline
\end{tabular}


TABLE 1: Continued.

\begin{tabular}{|c|c|c|c|c|c|c|}
\hline \multirow{2}{*}{ City/town } & \multicolumn{2}{|c|}{1985} & \multicolumn{2}{|c|}{1996} & \multicolumn{2}{|c|}{2005} \\
\hline & Area $A$ & Perimeter $P$ & Area $A$ & Perimeter $P$ & Area $A$ & Perimeter $P$ \\
\hline Yuyao & 14.40 & 64.56 & 15.69 & 87.89 & 63.83 & 157.04 \\
\hline Cixi & 4.67 & 24.09 & 17.69 & 54.75 & 67.35 & 146.38 \\
\hline Fenghua & 3.29 & 18.32 & 8.91 & 34.51 & 18.95 & 82.31 \\
\hline Jiaxing & 11.71 & 46.17 & 19.47 & 45.62 & 67.29 & 98.29 \\
\hline Jiashan & 4.31 & 22.66 & 4.72 & 13.57 & 23.27 & 45.71 \\
\hline Haiyan & 3.97 & 22.85 & 6.09 & 20.09 & 21.49 & 29.12 \\
\hline Haining & 5.64 & 22.75 & 8.27 & 24.67 & 23.34 & 57.09 \\
\hline Pinghu & 3.04 & 15.19 & 6.26 & 21.08 & 24.78 & 40.35 \\
\hline Tongxiang & 3.29 & 18.35 & 5.55 & 20.27 & 34.34 & 43.68 \\
\hline Huzhou & 10.43 & 28.61 & 14.19 & 31.84 & 30.15 & 50.59 \\
\hline Deqing & 1.49 & 5.77 & 3.05 & 9.87 & 22.52 & 33.88 \\
\hline Changxing & 6.81 & 30.88 & 7.34 & 30.28 & 23.24 & 43.68 \\
\hline Anji & 2.14 & 13.21 & 3.53 & 18.39 & 23.14 & 50.29 \\
\hline Shaoxing & 20.21 & 49.00 & 23.08 & 64.55 & 57.23 & 142.19 \\
\hline Chengxian & 3.38 & 16.11 & 7.59 & 22.36 & 8.08 & 28.06 \\
\hline Xinchang & 5.87 & 24.32 & 6.75 & 23.01 & 7.86 & 28.05 \\
\hline Zhuji & 3.57 & 24.14 & 12.82 & 55.66 & 65.86 & 110.29 \\
\hline Shangyu & 1.68 & 13.67 & 7.95 & 42.16 & 28.77 & 72.33 \\
\hline Zhoushan & 7.28 & 46.57 & 8.68 & 42.44 & 14.76 & 47.78 \\
\hline
\end{tabular}

TABle 2: The global fractal parameters of the cities and towns in Yangtze River Delta, China (1985-2005).

\begin{tabular}{lcccccc}
\hline \multirow{2}{*}{ Year } & \multicolumn{2}{c}{ Original results } & \multicolumn{2}{c}{ Revised results } \\
& $k$ & $b$ & $D_{l}=2 b$ & $R^{2}$ & $D_{b}=\left(1+D_{l}\right) / 2$ & 1.1963 \\
$D_{f}=1+1 / D_{l}$ \\
\hline 1985 & 8.0340 & 0.6963 & 1.3926 & 0.9282 & 1.2387 & 1.7181 \\
1996 & 6.1128 & 0.7387 & 1.4774 & 0.9144 & 1.2266 & 1.6769 \\
2005 & 5.4610 & 0.7266 & 1.4532 & 0.8879 & 1.2205 & 1.6944 \\
\hline Average & 6.5359 & 0.7205 & 1.4411 & 0.9102 & \\
\hline
\end{tabular}

the fractal measure relation indicates some disorder problems in the processes of urbanization and urban evolution. If the power law degenerates into a linear relation, we can treat it as quasiallometric scaling in light of fractal theory in order to bring the parameter values into comparison (Table 4).

Second, both the boundary dimension and the form dimension approach certain constants. This seems to suggest some conversation law of fractal parameter evolution. The average revised boundary dimension approaches a constant: $D_{b}=1.182$ for $1985, D_{b}=1.236$ for 1996 , and $D_{b}=1.227$ for 2005. In short, the mean of the textual dimension is close to 1.2 , namely, $D_{b} \rightarrow 1.2$. Accordingly, the average form dimension approaches another constant: $D_{f}=1.707$ for 1985, $D_{f}=1.693$ for 1996 , and $D_{f}=1.696$ for 2005 . In short, the mean of the structural dimension is close to 1.7 , namely, $D_{f} \rightarrow 1.7$ (Table 4 ). The average value of the form dimension lends further support to the suggestion that the structural dimension of urban space changes around $1.7[4,23,48]$. The $D_{f}=1.7$ is an interesting value for the form dimension of cities. By computer simulation, the fractal dimension of spatial aggregates such as cities proved to approach 1.7 averagely [50]; by empirical analysis, the form dimension mean of cities is near 1.7 [4]. It has been demonstrated that the reasonable range of the form dimension comes between 1.5 and 2 [48]. If $D_{f}<1.5$, the urban field is not efficiently utilized due to underfilling of space; on the contrary, if $D_{f}>$ 1.9 , the urban field is excessively utilized owing to overfilling of space. In theory, the best value of the form dimension is close to $1.7[23,35]$. In terms of $(8)$, the corresponding boundary dimension is about $D_{b}=1.2$.

Third, the fractal models and the fractal parameters reflect the social and economic state of China. For a long time, China is socialist country based on centrally planned economy rather than market economy. Chinese city development is always associated with its political and economic conditions. Since the introduction of the economic reforms and opening-up policies at the end of 1978 and with the gradual establishment of a socialist market economic system from 1992, namely, after Deng's South Tour Speeches, the command economy based on top-down mechanism and the market economy based on bottom-up mechanism combined with one another to form a mixed economy (Table 6). China has witnessed a rapid urban development [51]. If we relate the urban evolution with the political and economic background 
TABLE 3: The local fractal parameters of the cities and towns in Yangtze River Delta, China (1985-2005).

\begin{tabular}{|c|c|c|c|c|c|c|c|c|c|c|c|c|}
\hline \multirow{2}{*}{ City } & \multicolumn{4}{|c|}{1985} & \multicolumn{4}{|c|}{1996} & \multicolumn{4}{|c|}{2005} \\
\hline & $D_{l}$ & $D_{b}$ & $D_{f}$ & $\mathrm{Co}^{* *}$ & $D_{l}$ & $D_{b}$ & $D_{f}$ & Co & $D_{l}$ & $D_{b}$ & $D_{f}$ & Co \\
\hline Shanghai & 1.247 & 1.123 & 1.802 & 0.205 & 1.429 & 1.214 & 1.700 & 0.144 & 1.468 & 1.234 & 1.681 & 0.120 \\
\hline Qingpu & 1.728 & 1.364 & 1.579 & 0.242 & 1.416 & 1.208 & 1.706 & 0.364 & 1.465 & 1.233 & 1.683 & 0.304 \\
\hline Chongming & 1.405 & 1.203 & 1.711 & 0.320 & 1.434 & 1.217 & 1.697 & 0.389 & 1.424 & 1.212 & 1.702 & 0.401 \\
\hline Songjiang & 1.427 & 1.213 & 1.701 & 0.256 & 1.078 & 1.039 & 1.928 & 0.521 & 1.277 & 1.139 & 1.783 & 0.359 \\
\hline Jinshan & 1.261 & 1.130 & 1.793 & 0.363 & 1.460 & 1.230 & 1.685 & 0.388 & 1.125 & 1.063 & 1.889 & 0.528 \\
\hline Nanjing & 1.511 & 1.256 & 1.662 & 0.132 & 1.454 & 1.227 & 1.688 & 0.181 & 1.511 & 1.256 & 1.662 & 0.150 \\
\hline Jiangning & 1.230 & 1.115 & 1.813 & 0.349 & 1.567 & 1.283 & 1.638 & 0.257 & 1.510 & 1.255 & 1.662 & 0.250 \\
\hline Jiangpu & 1.851 & 1.426 & 1.540 & 0.253 & 1.969 & 1.484 & 1.508 & 0.208 & 1.617 & 1.308 & 1.619 & 0.277 \\
\hline Liuhe & 1.333 & 1.166 & 1.750 & 0.341 & 1.403 & 1.201 & 1.713 & 0.382 & 1.330 & 1.165 & 1.752 & 0.410 \\
\hline Lishui & 1.497 & 1.248 & 1.668 & 0.253 & 1.622 & 1.311 & 1.616 & 0.288 & 1.504 & 1.252 & 1.665 & 0.301 \\
\hline Gaochun & 1.168 & 1.084 & 1.856 & 0.389 & 1.533 & 1.266 & 1.652 & 0.389 & 1.598 & 1.299 & 1.626 & 0.314 \\
\hline Wuxi & 1.435 & 1.217 & 1.697 & 0.181 & 1.357 & 1.178 & 1.737 & 0.256 & 1.423 & 1.212 & 1.703 & 0.225 \\
\hline Jiangyin & 1.285 & 1.143 & 1.778 & 0.298 & 1.492 & 1.246 & 1.670 & 0.261 & 1.579 & 1.289 & 1.633 & 0.174 \\
\hline Yixing & 1.584 & 1.292 & 1.631 & 0.274 & 1.494 & 1.247 & 1.669 & 0.318 & 1.462 & 1.231 & 1.684 & 0.280 \\
\hline Changzhou & 1.422 & 1.211 & 1.703 & 0.183 & 1.489 & 1.245 & 1.672 & 0.182 & 1.473 & 1.236 & 1.679 & 0.184 \\
\hline Liyang & 1.543 & 1.272 & 1.648 & 0.307 & 1.749 & 1.375 & 1.572 & 0.224 & 1.676 & 1.338 & 1.597 & 0.240 \\
\hline Jintan & 1.506 & 1.253 & 1.664 & 0.298 & 1.613 & 1.306 & 1.620 & 0.279 & 1.443 & 1.221 & 1.693 & 0.336 \\
\hline Suzhou & 1.424 & 1.212 & 1.702 & 0.189 & 1.422 & 1.211 & 1.703 & 0.218 & 1.324 & 1.162 & 1.755 & 0.276 \\
\hline Changshu & 1.712 & 1.356 & 1.584 & 0.183 & 1.474 & 1.237 & 1.678 & 0.270 & 1.549 & 1.275 & 1.646 & 0.212 \\
\hline Zhangjiagang & 1.628 & 1.314 & 1.614 & 0.243 & 1.408 & 1.204 & 1.710 & 0.320 & 1.423 & 1.211 & 1.703 & 0.264 \\
\hline Kunshan & 1.584 & 1.292 & 1.631 & 0.233 & 1.272 & 1.136 & 1.786 & 0.389 & 1.298 & 1.149 & 1.770 & 0.350 \\
\hline Wujiang & 1.355 & 1.177 & 1.738 & 0.317 & 1.213 & 1.107 & 1.824 & 0.465 & 1.257 & 1.129 & 1.795 & 0.445 \\
\hline Taicang & 1.469 & 1.235 & 1.681 & 0.274 & 1.300 & 1.150 & 1.769 & 0.403 & 1.270 & 1.135 & 1.787 & 0.396 \\
\hline Nantong & 1.143 & 1.071 & 1.875 & 0.348 & 1.498 & 1.249 & 1.667 & 0.222 & 1.571 & 1.285 & 1.637 & 0.168 \\
\hline Hai'an & 1.270 & 1.135 & 1.787 & 0.320 & 1.312 & 1.156 & 1.762 & 0.389 & 1.382 & 1.191 & 1.724 & 0.339 \\
\hline Rudong & 1.017 & 1.008 & 1.984 & 0.436 & 1.178 & 1.089 & 1.849 & 0.488 & 1.317 & 1.158 & 1.759 & 0.426 \\
\hline Haimen & 0.981 & 0.991 & 2.019 & 0.449 & 1.394 & 1.197 & 1.717 & 0.389 & 1.536 & 1.268 & 1.651 & 0.311 \\
\hline Qidong & 0.950 & 0.975 & 2.053 & 0.462 & 1.251 & 1.125 & 1.800 & 0.443 & 1.491 & 1.246 & 1.671 & 0.310 \\
\hline Rugao & 1.498 & 1.249 & 1.667 & 0.285 & 1.675 & 1.338 & 1.597 & 0.230 & 1.445 & 1.222 & 1.692 & 0.322 \\
\hline Yangzhou & 1.448 & 1.224 & 1.691 & 0.195 & 1.703 & 1.351 & 1.587 & 0.153 & 1.544 & 1.272 & 1.647 & 0.200 \\
\hline Jiangdu & 1.267 & 1.134 & 1.789 & 0.336 & 1.634 & 1.317 & 1.612 & 0.276 & 1.599 & 1.299 & 1.625 & 0.232 \\
\hline Yizheng & 1.640 & 1.320 & 1.610 & 0.220 & 1.402 & 1.201 & 1.713 & 0.342 & 1.375 & 1.187 & 1.727 & 0.357 \\
\hline Taizhou & 1.382 & 1.191 & 1.724 & 0.255 & 1.532 & 1.266 & 1.653 & 0.256 & 1.613 & 1.306 & 1.620 & 0.176 \\
\hline Jiangyan (Tai) & 1.364 & 1.182 & 1.733 & 0.314 & 1.690 & 1.345 & 1.592 & 0.275 & 1.673 & 1.336 & 1.598 & 0.220 \\
\hline Taixing & 1.406 & 1.203 & 1.711 & 0.280 & 1.396 & 1.198 & 1.716 & 0.339 & 1.573 & 1.286 & 1.636 & 0.262 \\
\hline Jingjiang & 1.515 & 1.257 & 1.660 & 0.238 & 1.187 & 1.094 & 1.842 & 0.447 & 1.597 & 1.298 & 1.626 & 0.240 \\
\hline Zhenjiang & 1.539 & 1.270 & 1.650 & 0.177 & 1.610 & 1.305 & 1.621 & 0.182 & 1.677 & 1.338 & 1.596 & 0.156 \\
\hline Jurong & 1.842 & 1.421 & 1.543 & 0.259 & 1.579 & 1.290 & 1.633 & 0.334 & 1.569 & 1.285 & 1.637 & 0.336 \\
\hline Yangzhong & 1.340 & 1.170 & 1.746 & 0.354 & 1.376 & 1.188 & 1.727 & 0.411 & 1.696 & 1.348 & 1.590 & 0.271 \\
\hline Danyang & 1.583 & 1.291 & 1.632 & 0.247 & 1.434 & 1.217 & 1.698 & 0.322 & 1.439 & 1.219 & 1.695 & 0.301 \\
\hline Hangzhou & 1.423 & 1.212 & 1.703 & 0.181 & 1.532 & 1.266 & 1.653 & 0.169 & 1.328 & 1.164 & 1.753 & 0.272 \\
\hline Tonglu & 1.308 & 1.154 & 1.764 & 0.397 & 1.636 & 1.318 & 1.611 & 0.419 & 1.709 & 1.355 & 1.585 & 0.319 \\
\hline Fuyang & 1.253 & 1.127 & 1.798 & 0.385 & 1.680 & 1.340 & 1.595 & 0.288 & 1.358 & 1.179 & 1.736 & 0.316 \\
\hline Lin'an & 1.730 & 1.365 & 1.578 & 0.269 & 1.612 & 1.306 & 1.620 & 0.331 & 1.331 & 1.165 & 1.751 & 0.409 \\
\hline Yuhang & 1.305 & 1.152 & 1.766 & 0.416 & 1.537 & 1.269 & 1.651 & 0.358 & 1.393 & 1.197 & 1.718 & 0.368 \\
\hline Xiaoshan & 1.206 & 1.103 & 1.830 & 0.374 & 1.728 & 1.364 & 1.579 & 0.208 & 1.525 & 1.263 & 1.656 & 0.195 \\
\hline Ningbo & 1.374 & 1.187 & 1.728 & 0.234 & 1.423 & 1.211 & 1.703 & 0.253 & 1.402 & 1.201 & 1.713 & 0.225 \\
\hline Xiangshan & 1.513 & 1.256 & 1.661 & 0.317 & 1.341 & 1.170 & 1.746 & 0.495 & 1.299 & 1.149 & 1.770 & 0.482 \\
\hline Ninghai & 1.076 & 1.038 & 1.930 & 0.413 & 1.299 & 1.149 & 1.770 & 0.431 & 1.141 & 1.070 & 1.877 & 0.543 \\
\hline
\end{tabular}


TABLE 3: Continued.

\begin{tabular}{|c|c|c|c|c|c|c|c|c|c|c|c|c|}
\hline \multirow{2}{*}{ City } & \multicolumn{4}{|c|}{1985} & \multicolumn{4}{|c|}{1996} & \multicolumn{4}{|c|}{2005} \\
\hline & $D_{l}$ & $D_{b}$ & $D_{f}$ & $\mathrm{Co}^{* *}$ & $D_{l}$ & $D_{b}$ & $D_{f}$ & Co & $D_{l}$ & $D_{b}$ & $D_{f}$ & Co \\
\hline Yuyao & 1.563 & 1.281 & 1.640 & 0.208 & 1.937 & 1.468 & 1.516 & 0.160 & 1.616 & 1.308 & 1.619 & 0.180 \\
\hline Cixi & 1.425 & 1.213 & 1.702 & 0.318 & 1.526 & 1.263 & 1.655 & 0.272 & 1.562 & 1.281 & 1.640 & 0.199 \\
\hline Fenghua & 1.384 & 1.192 & 1.722 & 0.351 & 1.583 & 1.291 & 1.632 & 0.307 & 1.844 & 1.422 & 1.542 & 0.187 \\
\hline Jiaxing & 1.421 & 1.211 & 1.704 & 0.263 & 1.354 & 1.177 & 1.739 & 0.343 & 1.373 & 1.187 & 1.728 & 0.296 \\
\hline Jiashan & 1.420 & 1.210 & 1.704 & 0.325 & 1.028 & 1.014 & 1.973 & 0.568 & 1.350 & 1.175 & 1.741 & 0.374 \\
\hline Haiyan & 1.516 & 1.258 & 1.660 & 0.309 & 1.317 & 1.159 & 1.759 & 0.435 & 1.091 & 1.046 & 1.916 & 0.564 \\
\hline Haining & 1.203 & 1.102 & 1.831 & 0.370 & 1.321 & 1.160 & 1.757 & 0.413 & 1.490 & 1.245 & 1.671 & 0.300 \\
\hline Pinghu & 1.146 & 1.073 & 1.873 & 0.407 & 1.350 & 1.175 & 1.741 & 0.421 & 1.246 & 1.123 & 1.803 & 0.437 \\
\hline Tongxiang & 1.387 & 1.194 & 1.721 & 0.350 & 1.399 & 1.199 & 1.715 & 0.412 & 1.176 & 1.088 & 1.850 & 0.476 \\
\hline Huzhou & 1.083 & 1.042 & 1.923 & 0.400 & 1.244 & 1.122 & 1.804 & 0.419 & 1.307 & 1.154 & 1.765 & 0.385 \\
\hline Deqing* & -1.660 & -0.330 & 0.398 & 0.750 & 0.859 & 0.930 & 2.164 & 0.627 & 1.172 & 1.086 & 1.853 & 0.497 \\
\hline Changxing & 1.404 & 1.202 & 1.712 & 0.300 & 1.605 & 1.303 & 1.623 & 0.317 & 1.322 & 1.161 & 1.756 & 0.391 \\
\hline Anji & 1.307 & 1.154 & 1.765 & 0.393 & 1.747 & 1.373 & 1.573 & 0.362 & 1.413 & 1.207 & 1.708 & 0.339 \\
\hline Shaoxing & 1.203 & 1.101 & 1.831 & 0.325 & 1.502 & 1.251 & 1.666 & 0.264 & 1.611 & 1.305 & 1.621 & 0.189 \\
\hline Chengxian & 1.143 & 1.071 & 1.875 & 0.405 & 1.280 & 1.140 & 1.781 & 0.437 & 1.567 & 1.283 & 1.638 & 0.359 \\
\hline Xinchang & 1.252 & 1.126 & 1.799 & 0.353 & 1.388 & 1.194 & 1.720 & 0.400 & 1.587 & 1.294 & 1.630 & 0.354 \\
\hline Zhuji & 1.729 & 1.365 & 1.578 & 0.277 & 1.732 & 1.366 & 1.577 & 0.228 & 1.435 & 1.218 & 1.697 & 0.261 \\
\hline Shangyu & 2.049 & 1.525 & 1.488 & 0.336 & 1.863 & 1.431 & 1.537 & 0.237 & 1.538 & 1.269 & 1.650 & 0.263 \\
\hline Zhoushan & 1.770 & 1.385 & 1.565 & 0.205 & 1.793 & 1.397 & 1.558 & 0.246 & 1.611 & 1.306 & 1.621 & 0.285 \\
\hline Average & 1.364 & 1.182 & 1.707 & 0.307 & 1.472 & 1.236 & 1.693 & 0.330 & 1.454 & 1.227 & 1.696 & 0.307 \\
\hline
\end{tabular}

Note. ${ }^{*}$ The results of Deqing in 1985 are outliers. ${ }^{* *}$ The symbol "Co" denotes compactness ratio of urban form.

TABLE 4: A comparison between the global fractal parameters and the average local fractal parameters of the cities and towns in Yangtze River Delta, China (1985-2005).

\begin{tabular}{lccccc}
\hline \multirow{2}{*}{ Year } & \multicolumn{3}{c}{ Global estimation } & \multicolumn{3}{c}{ Local average } \\
& $D_{l}$ & $D_{b}$ & $D_{f}$ & $D_{l}$ & $D_{b}$ \\
\hline 1985 & 1.3926 & 1.1963 & 1.7181 & 1.3636 & 1.1818 \\
1996 & 1.4774 & 1.2387 & 1.6769 & 1.4722 & 1.2361 \\
2005 & 1.4532 & 1.2266 & 1.6882 & 1.4545 & 1.2272 \\
\hline Average & 1.4411 & 1.2205 & 1.6944 & 1.4301 & 1.6933 \\
\hline
\end{tabular}

TABLE 5: Comparisons between the $R$ square values of four possible models for the area-perimeter relationships of the cities and towns in Yangtze River Delta, China (1985-2005).

\begin{tabular}{lcccc}
\hline Year & Linear model & Exponential model & Logarithmic model & Power model \\
\hline 1985 & 0.747 & 0.372 & 0.793 & $\mathbf{0 . 9 2 8}$ \\
1996 & $\mathbf{0 . 9 1 9}$ & 0.441 & 0.722 & 0.914 \\
2005 & $\mathbf{0 . 9 3 9}$ & 0.439 & 0.626 & 0.888 \\
\hline
\end{tabular}

of China, we can understand the changes of fractals and fractal dimension of Chinese cities. At the beginning of reform and opening up of China, that is, in 1985, the fractal dimension values are in disorder to some extent, which can be reflected by the relation between the boundary dimension and compactness ratio (see Figure 3 and Section 4). Moreover, several values are abnormal: the $D_{b}$ value is less than 1 or even less than 0 , and the $D_{f}$ value is greater than 2 . The $D_{b}$ values range from -0.330 (an abnormal value) to 1.525 , and $D_{f}$ values vary from 0.398 to 2.053 . However, in 2005 , both the $D_{b}$ and $D_{f}$ values fluctuate between 1 and 2 . The $D_{b}$ values range from 1.046 to 1.422 , and the $D_{f}$ values vary from 1.542 to 1.916 (Table 3 ). The boundary dimension of cities is proved to be a function of the compactness of urban form. The fractal 
TABLE 6: Important historical events associated with urban evolution of China.

\begin{tabular}{|c|c|c|c|}
\hline Chance/change & Time & Mark/event & Consequence \\
\hline $\begin{array}{l}\text { Chinese economic reform and } \\
\text { open-up }\end{array}$ & $\begin{array}{l}1978-12-18 \\
1978-12-22 \\
\end{array}$ & $\begin{array}{l}\text { Chinese eleventh CPC Central } \\
\text { Committee Third Plenary Session }\end{array}$ & $\begin{array}{c}\text { Close economic systems change to } \\
\text { open systems }\end{array}$ \\
\hline $\begin{array}{l}\text { The socialist market economic } \\
\text { system }\end{array}$ & $\begin{array}{l}1992-1-18 \\
1992-2-21 \\
\end{array}$ & Deng's South Tour Speeches & Self-organized economics appears \\
\hline $\begin{array}{l}\text { Further economic reform and } \\
\text { open-up }\end{array}$ & 2001-12-11 & $\begin{array}{l}\text { Joining World Trade Organization } \\
\text { (WTO) }\end{array}$ & $\begin{array}{l}\text { Introduced international rules into } \\
\text { open economic systems }\end{array}$ \\
\hline
\end{tabular}

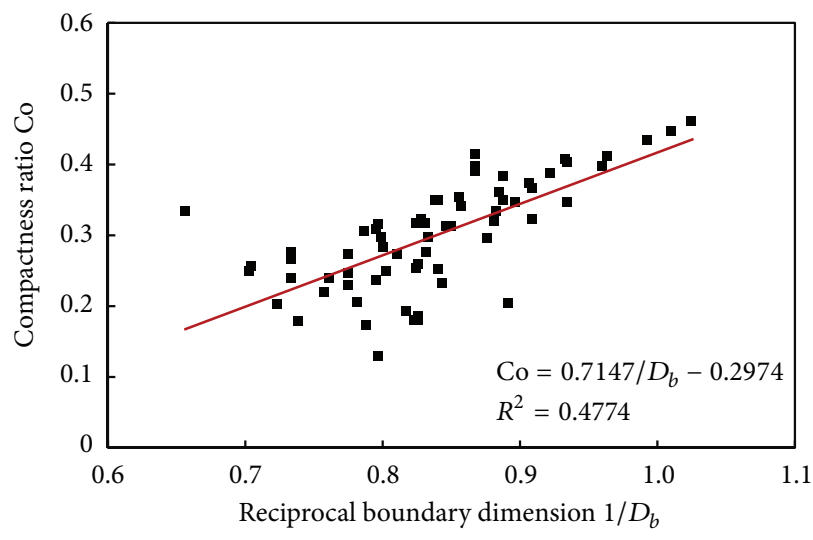

(a) 1985

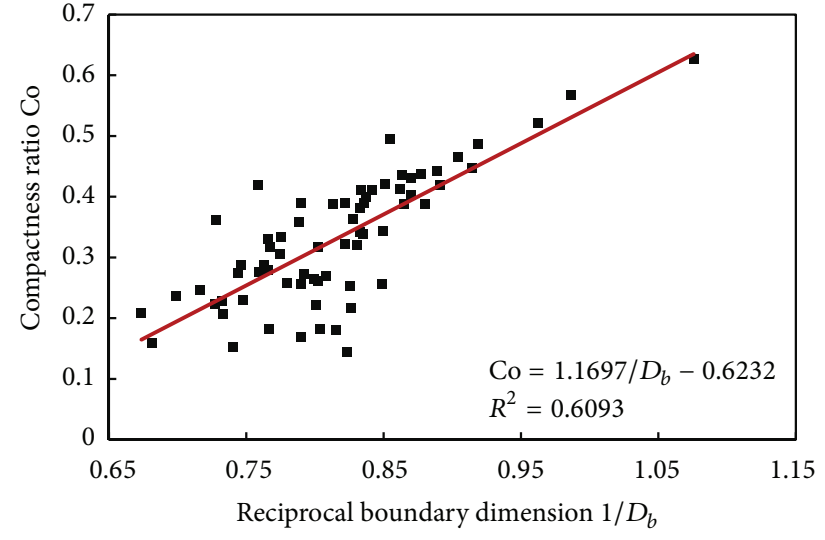

(b) 1996

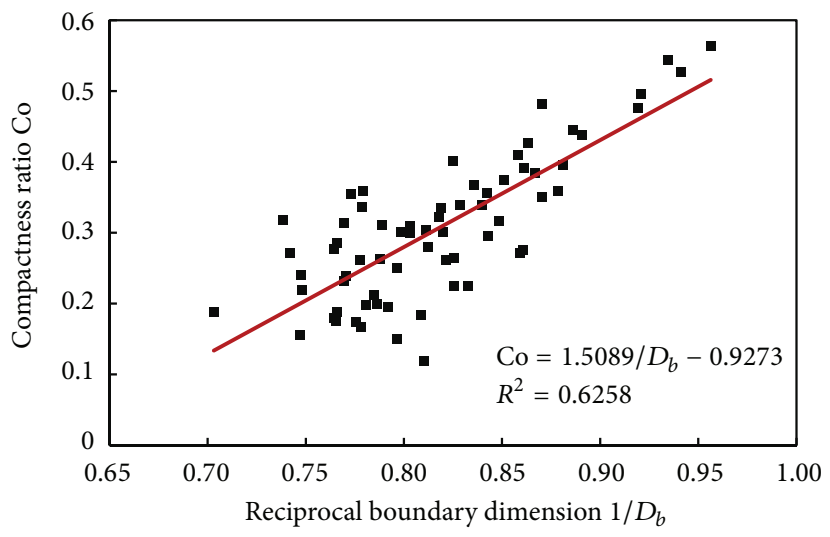

(c) 2005

FIgURE 3: The relationships between reciprocal of the boundary dimension and the compactness ratio of the cities and towns in Yangtze River Delta, China (1985-2005) [note: for 1985, the fractal dimension of Deqing is less than 0 and is removed as an outlier].

dimension evolution from chaos to order can be mirrored by the relations between the boundary dimension and the compactness ratio (Figure 3 ).

\section{Questions and Discussion}

An urban analytical process based on the area-perimeter scaling is illustrated by using the 68 cities and towns in the study area. Through the empirical analysis, the following questions have been clarified. First, the fractal dimension of urban shape includes form dimension and boundary dimension, which are associated with one another. However, the boundary dimension of cities and patches of a city are indeed overestimated by using the traditional area-perimeter scaling formula. In Table 3, many $D_{l}$ values are close to 2 , and this is unreasonable because the dimension of a fractal boundary is often less than $3 / 2$ [48]. By the ideas from multifractals and spatial correlation, we can demonstrate that $D=1.5$ is a threshold value of urban shape dimension, and there is a reasonable numerical range for the form dimension; that is, $1.5 \leq D_{f}<2$ [48]. However, in previous literature, the boundary dimension is often overestimated using (1). Thus, the boundary dimension used to be expressed as $D_{l}=b d=2 b$. Here $d=2$ represents the Euclidean dimension of urban region. This suggests that the urban area is regarded as a 2-dimensional measure. However, the average dimension of urban area in a system is not actually equal to $2[12,23,42,44]$. According to (5), the revised boundary 


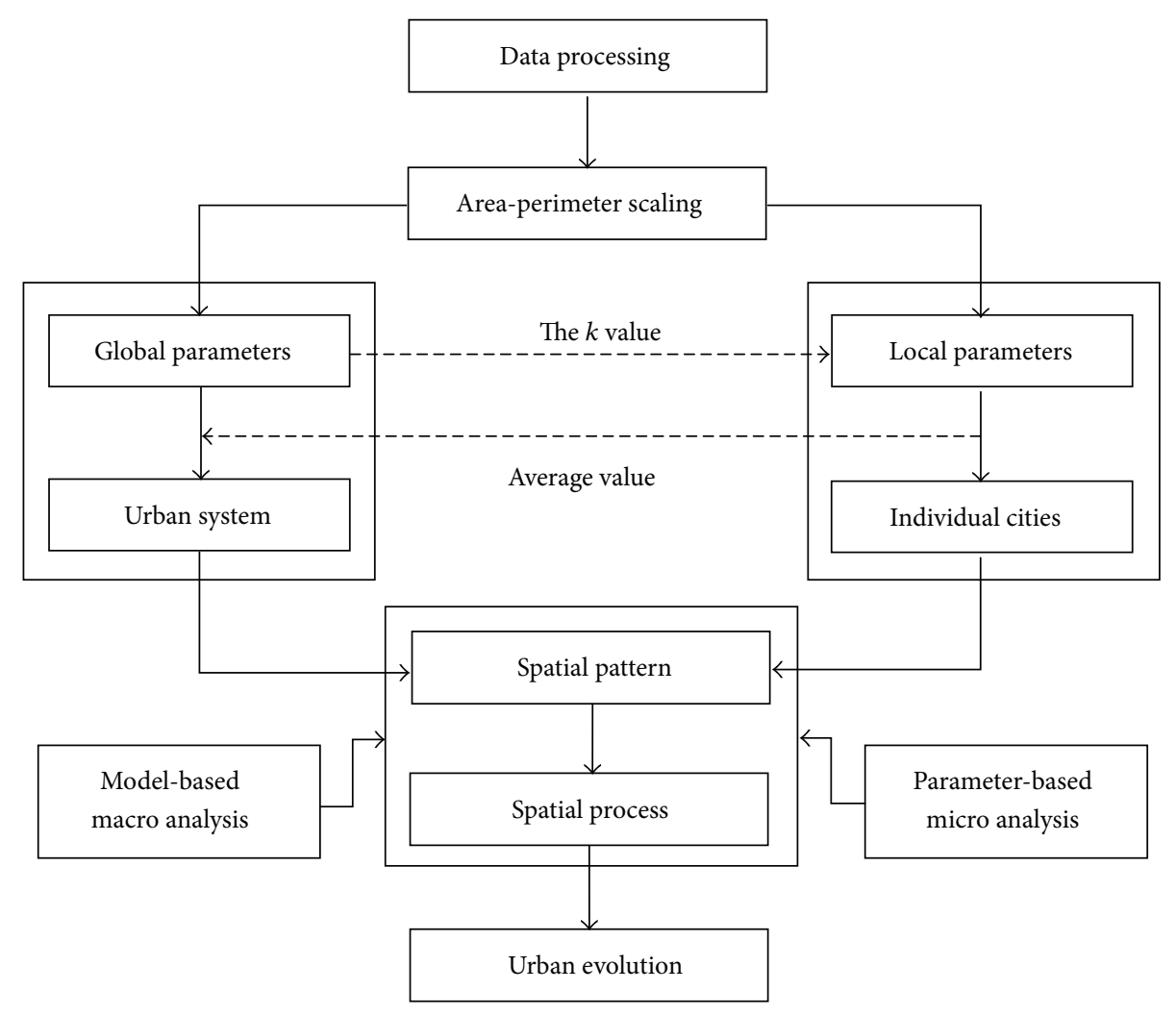

FIGURE 4: A schematic diagram of the urban analytical process based on the area-perimeter scaling.

dimension can be expressed as $D_{b}=\sigma D_{f}$, where the form dimension is near 1.7 where average is concerned $[4,30]$. Second, using the adjusting formula of fractal dimension, we can not only revise the boundary dimension indicating urban texture but also estimate the form dimension indicative of urban structure. The two formulae were previously derived [23], but the systematic empirical analysis is made for the first time. Third, in terms of the area-perimeter scaling, the fractal dimensions of urban shape fall into two types: the global parameters indicative of a system of cities and the local parameter indicating the individual cities in the urban system. If we estimate the global parameters by using the least squares calculations, the proportionality coefficient values in the local fractal parameter formula can be obtained by the constants of the global models. In other words, if we estimate the $k$ values for an urban system by the regression analysis based on (1), we can compute the boundary dimension of each city in the urban system by substituting the $k$ value in (3). This implies that we can use a dynamics proportionality factor $k$ instead of a fixed coefficient $k=4$ to estimate the local fractal parameters of cities. The analytical process of fractal cities can be demonstrated by a block diagram (Figure 4).

The main limitation of this study lies in the resolution of the remote sensing images. The resolution of the remote sensing images of the cities and towns is not high enough to guarantee the spatial data quality of individual cities. In this case, we cannot abstract large datasets of urban area and perimeter based on different scales for each human settlement. For a city, we have only two data points: urban area and perimeter. As a result, we cannot calculate the boundary dimension and form dimension for individual cities directly. In other words, we cannot evaluate the local fractal parameters for individual cities by the least squares calculation due to absence of datasets for each city. What we can do is to evaluate the global fractal parameters for the urban systems by means of the area-perimeter scaling based on cross-sectional data and then estimate the local fractal parameters for each city by the approximate formula. The shortcomings of the empirical analysis are as below. First, there exist many outliers in the results. For example, the boundary dimension of Deqing city is negative, which is absurd as a fractal dimension must be greater than 0 and less than the Euclidean dimension of the embedding space. A fractal city based on the remote sensing images is actually defined in a 2-dimensional space [4]. Thus, the Euclidean dimension of the embedding space for the fractal city is $d=2$. The boundary dimension of Shangyu is greater than 2, going beyond the Euclidean dimension of its embedding space. Second, the comparability of the fractal dimension values in different year is doubtful. Generally speaking, owing to space filling, the form dimension increases year by year, until the limit is reached $\left(D_{\max }=d=2\right)$. However, what with the data quality and the urban sprawl, the form dimension values of the cities and towns randomly fluctuated. Third, the numerical relation between the boundary and the compactness ratio degenerated. The boundary dimension of 
a city can be associated with the compactness of its urban form. The mathematical relation between the compactness ratio and the boundary dimension can be derived as below [20]:

$$
\text { Co }=\frac{K}{P} \exp \left(\frac{1}{D_{p}} \ln P\right),
$$

where Co denotes the compactness ratio of urban form, $D_{p}$ represents the boundary dimension $\left(D_{l}\right.$ or $\left.D_{b}\right), P$ is the length of urban perimeter, and $K$ is a coefficient. However, based on the datasets in Table 3, the exponential form of (10) is replaced by a hyperbolic relation such as

$$
\mathrm{Co}=u+\frac{v}{D_{p}}
$$

where $u$ and $v$ represent the intercept and slope if the reciprocal of the fractal dimension, and $1 / D_{p}$ is treated as an independent variable. Despite these shortcomings, we can make use of the advantages and bypass the disadvantages of the remote sensing data in virtue of the formulae of the fractal dimension estimation.

A popular misunderstanding of the boundary dimension should be pointed out here. In previous literature, the initial boundary dimension $D_{l}$ is always associated with the stability index of urban evolution [52]. If the parameter $D_{l}=1.5$, the urban boundary is regarded as resulting from Brownian motion and thus the urban development is considered to be unstable. This viewpoint is wrong. The reasons are as follows. First, it is the self-affine record dimension rather than the self-similar trail dimension that can be directly related with Brownian motion [28]. However, the boundary dimension of urban shape is a self-similar trail dimension instead of a self-affine record dimension [35]. Second, as indicated above, the boundary dimension is always numerically overestimated by the old formula. In fact, the properly estimated boundary dimension is seldom greater than or equal to 1.5. An urban boundary can be treated as a fractal line, and the dimension of a fractal line is usually less than 1.5. The boundary dimension should be revised using the adjusting formula (6). The revised boundary dimension values often come between 1 and 1.5 [23]. Finally, a random process does not indicate an unstable process. If the self-affine record dimension is close to 1.5 , it suggests randomicity rather than instability of urban growth.

\section{Conclusions}

In this paper, a case study is employed to illustrate a systematic analytical process of fractal cities using the areaperimeter allometric scaling. In particular, we try to develop an approach to make the best use of the advantages and bypass the disadvantages of spatial data by means of the new fractal dimension formulae. The innovation of this paper is with two aspects: First, the global fractal parameters and the local fractal parameters are integrated to make urban spatial analyses, and the numerical link between the global and local parameters is revealed by statistical average. Second, the form dimension and the boundary dimension are associated with one another to describe urban shapes. Third, the area-perimeter scaling is employed to estimate the proportionality coefficient for the approximate formula of boundary dimension. An interesting finding is the numerical link between the global and local parameters.

Based on the empirical analysis using the methodology developed in this work, the main conclusions can be reached as follows. First, the relationships between urban area and perimeter follow the allometric scaling laws; the scaling exponent is the ratio of the urban boundary dimension to the urban form dimension. However, the boundary dimension value used to be overestimated because the form dimension $\left(D_{f}<2\right)$ is mistaken for Euclidean dimension of urban area $(d=2)$. By using the revision formulae of fractal dimension, we can correct the results of fractal dimension estimation. What is more, the form dimension can be estimated through the boundary dimension. Thus, the form dimension indicative of urban structure and the boundary dimension indicating urban texture can be combined with each other to characterize urban evolution. Second, a fractal system of cities should be characterized by both the global and the local scaling parameters. The fractal parameters of an urban system, including form dimension and boundary dimension, fall into two groups: global parameters and local parameters. The global parameters reflect the spatial properties of a system of cities, while the local parameters reflect the geographical feature of individual cities. The average values of the local parameters are approximately equal to the corresponding values of the global parameters. This suggests that the global parameters can be decomposed into local parameters. By means of the average values, we can associate the global parameters with local parameters and further connect the macrolevel of an urban system with the microlevel of elements in the urban system. Third, the fractal measure relations between urban area and urban envelope may degenerate because of disorder competition of cities. Fractals follow scaling laws, which can be expressed as a set of power functions. However, a power-law relation between urban area and perimeter sometimes degenerates into a semilogarithmic relation or even a linear relation. Fractal structure based on power laws is a kind of optimized structure of natural and human systems. A fractal object can fill its space in the most efficient way. Fractal relation degeneration suggests some latent problems of urban evolution. If a city or urban system does not follow the allometric scaling law, the fractal structure may be broken and the structure of the city or system of cities should be improved by rational city planning.

\section{Conflict of Interests}

The authors declare that there is no conflict of interests regarding the publication of this paper.

\section{Acknowledgment}

This research was supported financially by the National Natural Science Foundation of China (Grant no. 41171129). The support is gratefully acknowledged. 


\section{References}

[1] G. Kane, "The mysteries of mass," Scientific American, vol. 293, no. 1, pp. 40-48, 2005.

[2] J. Henry, The Scientific Revolution and the Origins of Modern Science, Palgrave MacMillan, New York, NY, USA, 2nd edition, 2002.

[3] M. Batty, "The size, scale, and shape of cities," Science, vol. 319, no. 5864, pp. 769-771, 2008.

[4] M. Batty and P. A. Longley, Fractal Cities: A Geometry of Form and Function, Academic Press, London, UK, 1994.

[5] M.-L. De Keersmaecker, P. Frankhauser, and I. Thomas, "Using fractal dimensions for characterizing intra-urban diversity: the example of Brussels," Geographical Analysis, vol. 35, no. 4, pp. 310-328, 2003.

[6] P. Frankhauser, The Fractal Aspects of Urban Structures, Economica, Paris, France, 1994 (French).

[7] P. Frankhauser, "The fractal approach: a new tool for the spatial analysis of urban agglomerations," Population: An English Selection. New Methodological Approaches in the Social Sciences, vol. 10, no. 1, pp. 205-240, 1998.

[8] I. Thomas, P. Frankhauser, and M.-L. De Keersmaecker, "Fractal dimension versus density of built-up surfaces in the periphery of Brussels," Papers in Regional Science, vol. 86, no. 2, pp. 287308, 2007.

[9] I. Thomas, P. Frankhauser, B. Frenay, and M. Verleysen, "Clustering patterns of urban built-up areas with curves of fractal scaling behaviour," Environment and Planning B: Planning and Design, vol. 37, no. 5, pp. 942-954, 2010.

[10] M. Batty and Y. Xie, "Self-organized criticality and urban development," Discrete Dynamics in Nature and Society, vol. 3, no. 2-3, pp. 109-124, 1999.

[11] L. Benguigui, D. Czamanski, and M. Marinov, "The dynamics of urban morphology: the case of Petah Tikvah," Environment and Planning B: Planning and Design, vol. 28, no. 3, pp. 447-460, 2001.

[12] L. Benguigui, E. Blumenfeld-Lieberthal, and D. Czamanski, "The dynamics of the Tel Aviv morphology," Environment and Planning B: Planning and Design, vol. 33, no. 2, pp. 269-284, 2006.

[13] R. White, "Cities and cellular automata," Discrete Dynamics in Nature and Society, vol. 2, no. 2, pp. 111-125, 1998.

[14] L. Benguigui, D. Czamanski, M. Marinov, and Y. Portugali, "When and where is a city fractal?" Environment and Planning B: Planning and Design, vol. 27, no. 4, pp. 507-519, 2000.

[15] J. Feng and Y. G. Chen, "Spatiotemporal evolution of urban form and land-use structure in Hangzhou, China: evidence from fractals," Environment and Planning B: Planning and Design, vol. 37, no. 5, pp. 838-856, 2010.

[16] Y. G. Chen and J. J. Wang, "Multifractal characterization of urban form and growth: the case of Beijing," Environment and Planning B: Planning and Design, vol. 40, no. 5, pp. 884-904, 2013.

[17] G. Shen, "Fractal dimension and fractal growth of urbanized areas," International Journal of Geographical Information Science, vol. 16, no. 5, pp. 419-437, 2002.

[18] I. Thomas, P. Frankhauser, and C. Biernacki, "The morphology of built-up landscapes in Wallonia (Belgium): a classification using fractal indices," Landscape and Urban Planning, vol. 84, no. 2, pp. 99-115, 2008.
[19] R. White and G. Engelen, "Urban systems dynamics and cellular automata: fractal structures between order and chaos," Chaos, Solitons \& Fractals, vol. 4, no. 4, pp. 563-583, 1994.

[20] Y. Chen, "Derivation of the functional relations between fractal dimension of and shape indices of urban form," Computers, Environment and Urban Systems, vol. 35, no. 6, pp. 442-451, 2011.

[21] P. A. Longley, M. Batty, and J. Shepherd, "The size, shape and dimension of urban settlements," Transactions of the Institute of British Geographers, vol. 16, no. 1, pp. 75-94, 1991.

[22] M. Batty and P. A. Longley, "The morphology of urban land use," Environment and Planning B: Planning and Design, vol. 15, no. 4, pp. 461-488, 1988.

[23] Y. G. Chen, "A set of formulae on fractal dimension relations and its application to urban form," Chaos, Solitons \& Fractals, vol. 54, no. 1, pp. 150-158, 2013.

[24] P. A. Longley and M. Batty, "Fractal measurement and line generalization," Computers \& Geosciences, vol. 15, no. 2, pp. 167183, 1989.

[25] P. A. Longley and M. Batty, "On the fractal measurement of geographical boundaries," Geographical Analysis, vol. 21, no. 1, pp. 47-67, 1989.

[26] Y. Song, S. J. Wang, Q. Ye, and X. W. Wang, "Urban spatial morphology characteristic and its spatial differentiation of mining city in China," Areal Research and Development, vol. 31, no. 1, pp. 45-49, 2012 (Chinese).

[27] X. Wang, J. Liu, D. Zhuang, and L. Wang, "Spatial-temporal changes of urban spatial morphology in China," Acta Geographica Sinica, vol. 60, no. 3, pp. 392-400, 2005 (Chinese).

[28] J. Feder, Fractals, Plenum Press, New York, NY, USA, 1988.

[29] B. B. Mandelbrot, The Fractal Geometry of Nature, W. H. Freeman and Company, New York, NY, USA, 1983.

[30] Y. G. Chen, "Characterizing growth and form of fractal cities with allometric scaling exponents," Discrete Dynamics in Nature and Society, vol. 2010, Article ID 194715, 22 pages, 2010.

[31] M. Batty, R. Carvalho, A. Hudson-Smith, R. Milton, D. Smith, and P. Steadman, "Scaling and allometry in the building geometries of greater London," The European Physical Journal B: Condensed Matter and Complex Systems, vol. 63, no. 3, pp. 303-314, 2008.

[32] L. M. Bettencourt, “The origins of scaling in cities," Science, vol. 340, no. 6139, pp. 1438-1441, 2013.

[33] L. M. A. Bettencourt, J. Lobo, D. Helbing, C. Kühnert, and G. B. West, "Growth, innovation, scaling, and the pace of life in cities," Proceedings of the National Academy of Sciences of the United States of America, vol. 104, no. 17, pp. 7301-7306, 2007.

[34] J. Lobo, L. M. A. Bettencourt, D. Strumsky, and G. B. West, "Urban scaling and the production function for cities," PLoS ONE, vol. 8, no. 3, Article ID e58407, 2013.

[35] Y. G. Chen, "Exploring the fractal parameters of urban growth and form with wave-spectrum analysis," Discrete Dynamics in Nature and Society, vol. 2010, Article ID 974917, 20 pages, 2010.

[36] P. S. Addison, Fractals and Chaos: An Illustrated Course, Institute of Physics Publishing, Bristol, UK, 1997.

[37] B. H. Kaye, A Random Walk Through Fractal Dimensions, VCH, New York, NY, USA, 1989.

[38] P. Haggett, A. D. Cliff, and A. Frey, Locational Analysis in Human Geography, Arnold Publishers, London, UK, 2nd edition, 1977.

[39] H. Takayasu, Fractals in the Physical Sciences, Manchester University Press, Manchester, UK, 1990. 
[40] X. L. Chang, "The study of relationship between the process of desertification and the landscape pattern in Bashing region, Hebei Province," Journal of Desert Research, vol. 16, no. 3, pp. 222-227, 1996 (Chinese).

[41] X. L. Chang and J. G. Wu, "Spatial analysis of pattern of sandy landscapes in Kerqin, Inner Mongolia," Acta Ecologica Sinica, vol. 18, no. 3, pp. 225-232, 1998 (Chinese).

[42] Q. Cheng, “The perimeter-area fractal model and its application to geology," Mathematical Geology, vol. 27, no. 1, pp. 69-82, 1995.

[43] A. R. Imre, "Artificial fractal dimension obtained by using perimeter-area relationship on digitalized images," Applied Mathematics and Computation, vol. 173, no. 1, pp. 443-449, 2006.

[44] A. R. Imre and J. Bogaert, "The fractal dimension as a measure of the quality of habitats," Acta Biotheoretica, vol. 52, no. 1, pp. 41-56, 2004.

[45] Y. Chen and S. Jiang, "An analytical process of the spatiotemporal evolution of urban systems based on allometric and fractal ideas," Chaos, Solitons \& Fractals, vol. 39, no. 1, pp. 4964, 2009.

[46] H. Luo and Y. Chen, "An allometric algorithm for fractalbased Cobb-Douglas function of geographical systems," Discrete Dynamics in Nature and Society, vol. 2014, Article ID 910457, 10 pages, 2014.

[47] J. A. Richard, Remote Sensing Digital Image Analysis: An Introduction, Springer, Berlin, Germany, 5th edition, 2013.

[48] Y. G. Chen, "Fractal analytical approach of urban form based on spatial correlation function," Chaos, Solitons \& Fractals, vol. 49, no. 1, pp. 47-60, 2013.

[49] T. Chen, Studies on fractal systems of cities and towns in the central plains of China [M.S. thesis], Department of Geography, Northeast Normal University, Changchun, China, 1995 (Chinese).

[50] M. Batty, "Generating urban forms from diffusive growth," Environment \& Planning A, vol. 23, no. 4, pp. 511-544, 1991.

[51] D. L. Yang, Calamity and Reform in China: State, Rural Society, and Institutional Change since the Great Leap Famine, Stanford University Press, Redwood, Calif, USA, 1998.

[52] X. H. Zhu, Fractal and Fractal Dimensions of Spatial GeoInformation, Surveying and Mapping Press, Beijing, China, 2007 (Chinese). 


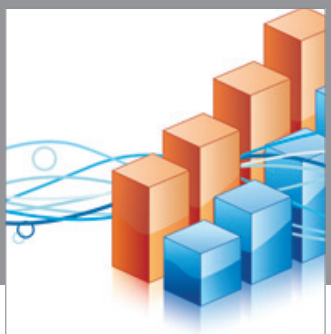

Advances in

Operations Research

vatem alat4

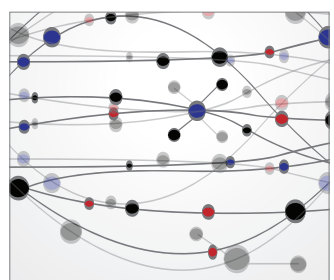

\section{The Scientific} World Journal
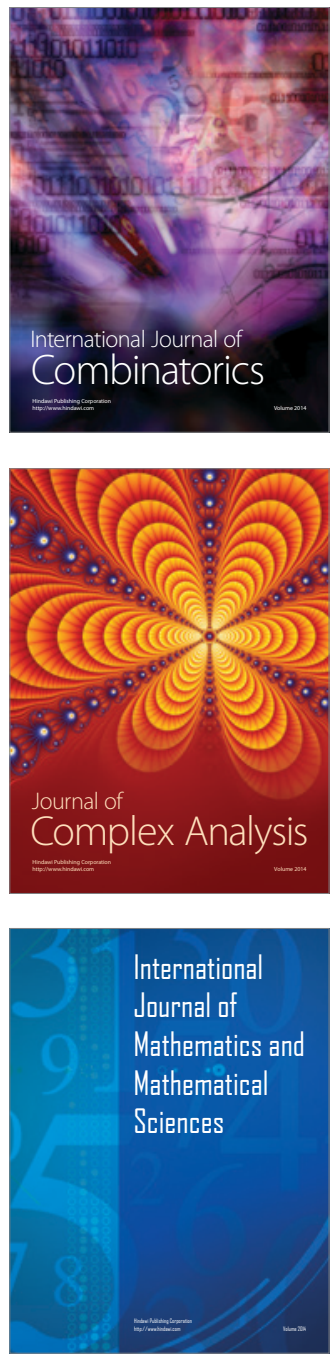
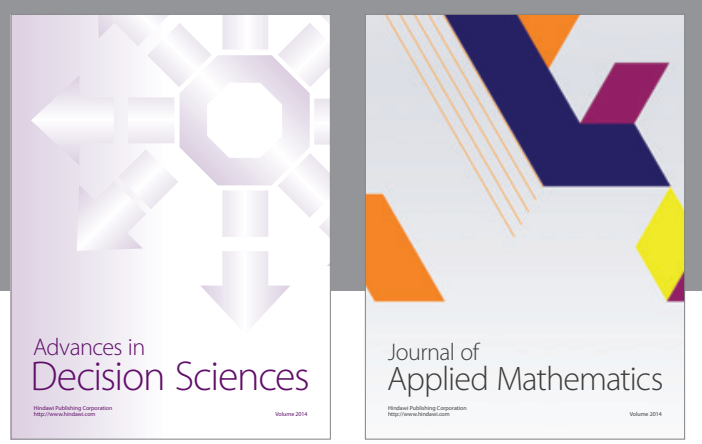

Algebra

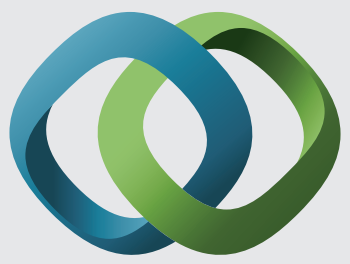

\section{Hindawi}

Submit your manuscripts at

http://www.hindawi.com
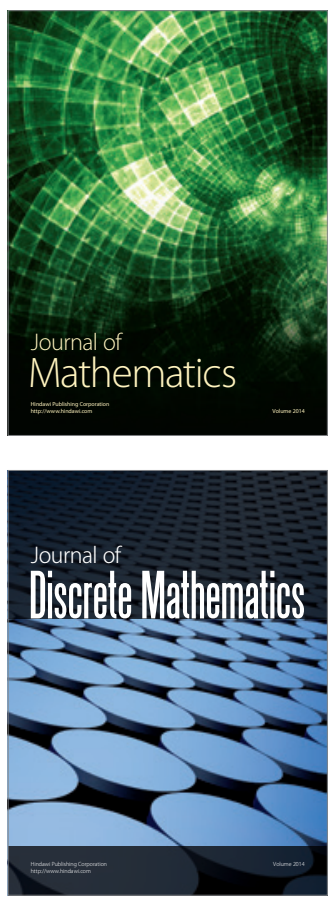

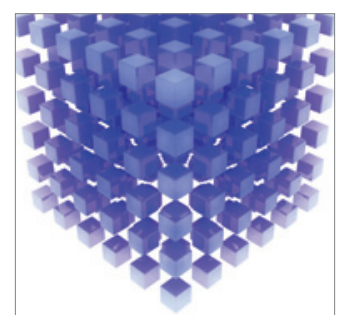

Mathematical Problems in Engineering
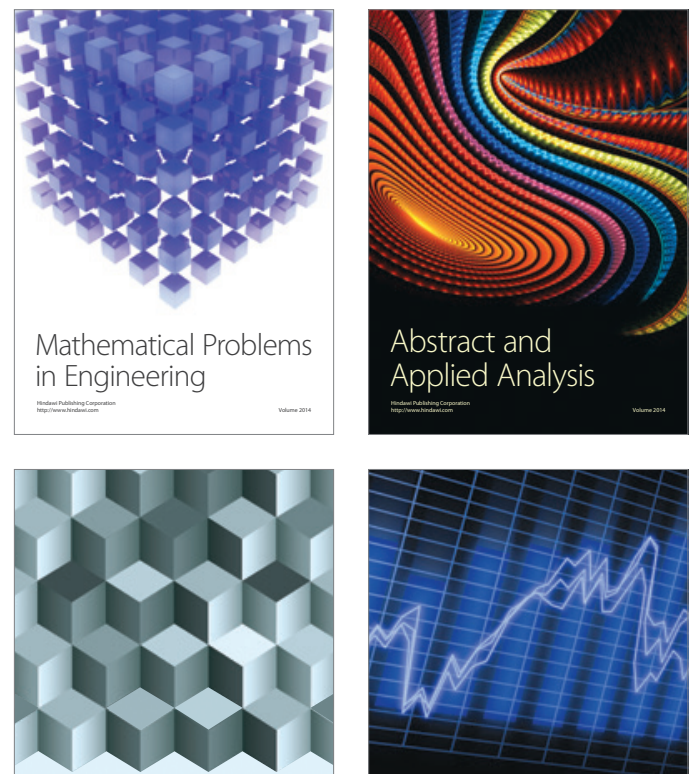

Journal of

Function Spaces

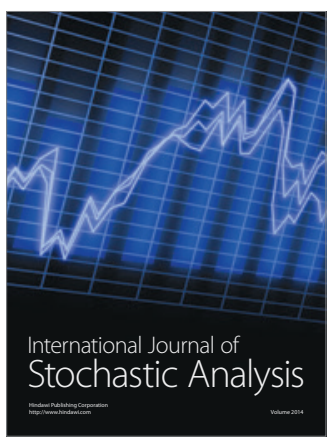

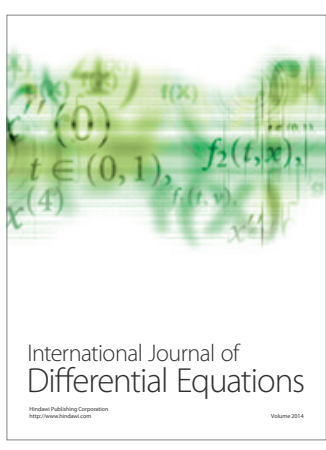
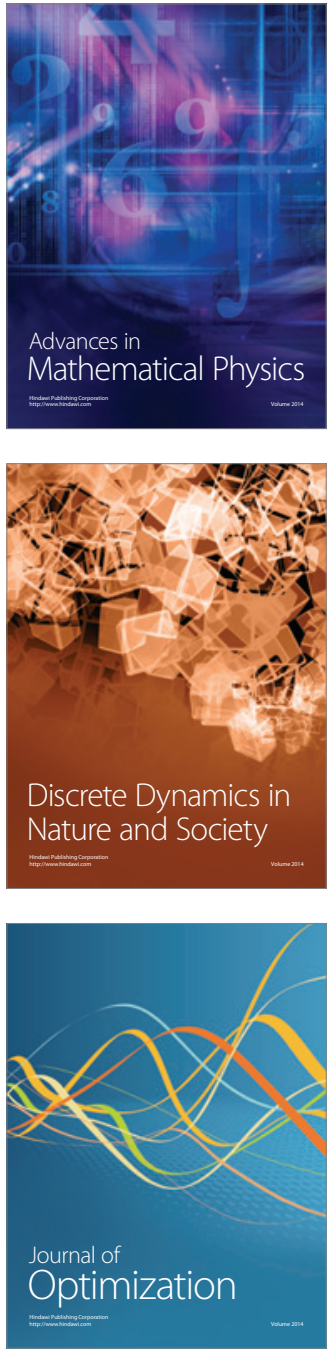